investigación económica, vol. LXX, 277, julio-septiembre de 2011, pp. 15-36

\title{
La naturaleza de una economía
}

Axel Leijonhufvud*

La crisis financiera y la recesión que se derivó de ella han propiciado una reexaminación del estado de la teoría macroeconómica. Las opiniones respecto de cuán serios son sus problemas difieren, pero tanto sus críticos como sus defensores coinciden en que deberían abordarse mediante un examen sistemático y, donde se considere necesario, modificar los supuestos de la teoría del equilibrio general dinámico estocástico (DSGE por sus siglas en inglés) dominante.

Esta es una reacción muy natural ante el fracaso conceptual. Pero ¿ंes la estrategia correcta?

- ¿Cuántos supuestos críticos requieren reexaminación?

- ¿Cuál es el marco dentro del cual identificamos los supuestos que parecen críticos?

- ¿Cómo contamos las inferencias de las cuales ni siquiera estamos advertidos?

Manuscrito recibido en marzo de 2011; aceptado en junio de 2011.

* Profesor Emérito de la University of California Los Angeles (UCLA) y Profesor de Teoría y Política Monetaria de la Università degli Studi di Trento, Italia, <stigaxel33@gmail.com>. Este artículo fue presentado en la conferencia de la Asociación Económica Argentina, Buenos Aires, que se verificó los días 15-19 de noviembre de 2010. Una versión anterior se presentó en la Conferencia de INET, Budapest del 6-8 de septiembre de 2010 y se publicó en inglés por el Centre for Policy Research, Policy Insight, no. 53, febrero de 2011. Dada la relevancia de las ideas y análisis contenidos en este trabajo, el autor del artículo y el Director-Editor de Investigación Económica convinieron en publicar una versión castellana para beneficio de los lectores latinoamericanos interesados en el tema. Agradezco a Ignacio Perrotini y a Estefanía Molerés por su impecable traducción al castellano y a los árbitros que dictaminaron esta versión. 
La macroeconomía al día de hoy tiene una larga historia en la que ha respondido a los problemas que asedian a la teoría prevaleciente en un momento determinado del tiempo cambiando uno o más de sus supuestos -y entonces continúa a partir de ahí.

Pero esta estrategia colectiva ahora parece habernos conducido a un desorden peor que nunca. ¿Podría ser que nuestros problemas consistan en algo más profundo?

\section{UNA MIRADA RETROSPECTIVA ${ }^{1}$}

En la Teoría General, Keynes propuso una teoría en la cual los salarios monetarios flexibles no restaurarían el pleno empleo en la economía, y salarios muy flexibles producirían una catástrofe financiera.

El modelo IS-LM, que se originó como un intento de formalizar la economía verbal de Keynes, después de años de debates condujo a la aparentemente inevitable conclusión de que el desempleo tenía que deberse a la inflexibilidad a la baja de los salarios monetarios. Así, esta vieja síntesis neoclásica interpreta la economía keynesiana como un sistema estable con una "fricción", más que como una teoría de una economía que alberga inestabilidades peligrosas.

Cuando la escala nominal de las economías occidentales se movió a la deriva sin dirección en los años sesenta y setenta, los modelos con salarios rígidos no fueron de mucha utilidad. La respuesta ad hoc fue adherir la curva de Phillips al modelo IS-LM. Ésta devino en el talón de Aquiles de la versión IS-LM de la economía keynesiana.

Al reformular la Teoría Cuantitativa en primeras diferencias, Friedman (1968) elaboró el instrumento de ataque perfecto, explicando al mismo tiempo la inestabilidad de la Curva de Phillips y la prima Fisher sobre las tasas de interés nominales. Al tiempo en que hacía esto, sin embargo, Friedman también introdujo el concepto de la tasa natural de desempleo.

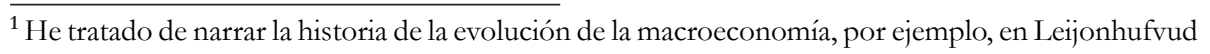
(1981 y 1992). 
En la teoría monetarista, la flexibilidad de los salarios era suficiente para garantizar que la economía convergiera a la tasa natural de desempleo -una doctrina que predominó en la macroeconomía durante 40 años.

En la teoría de Keynes esta proposición es falsa. Si el ahorro deseado no es igual a la inversión a la tasa natural de desempleo, la flexibilidad de los salarios no hará que converja a esa tasa. La teoría monetarista ignoró completamente el problema del ahorro y la inversión. Esto implicó también la omisión del papel de los mercados de crédito en alentar u obstruir la coordinación del ahorro y la inversión.

La principal tradición en la teoría de la política monetaria desde los días de Henry Thornton se había enfocado en la estabilización del crédito en los regímenes con dinero convertible. La doctrina de la política monetarista se preocupaba exclusivamente de la estabilización del nivel de precios. Ciertamente era natural dar prioridad a este objetivo en un momento en que los Estados Unidos estaban propagando los últimos vestigios de la convertibilidad del oro y desplazándose hacia un patrón fiduciario puro. Pero soslayando para el futuro -o, debiera decir, para el presente que estamos viviendo ahora- la inestabilidad potencial de los problemas acumulados del crédito.

El monetarismo friedmaniano no duró mucho. Robert Lucas creía en la teoría de Friedman, pero pensaba que carecía de microfundamentos. Lucas mostró cómo la inestabilidad de la curva de Phillips y la prima Fisher podían explicarse obedeciendo los dictados de la teoría de la elección óptima. Su modelo, sin embargo, tenía la propiedad de que sólo los cambios "no anticipados" en la tasa de crecimiento de la cantidad de dinero provocarían que el desempleo se desviara de su tasa natural.

Friedman no compartió este punto de vista. Después de no mucho tiempo se consideró como insostenible debido a una combinación de razones empíricas y teóricas. Pero esto no condujo al regreso de la economía friedmaniana. En vez de ello, el método analítico fundado por Lucas y sus colaboradores Nuevos Clásicos condujo a la macroeconomía por una dirección radicalmente diferente. 
El problema, tal como fue visto en su momento, era explicar las variaciones en el empleo sin recurrir al "dinero no anticipado" o, por supuesto, al problema del ahorro y la inversión que para entonces estaba olvidado. La respuesta, encabezada por Edward Prescott, fue la teoría de los ciclos económicos reales. En esta teoría, las variaciones en el producto y el empleo eran respuestas óptimas a variaciones exógenas (i.e. no explicadas) en el crecimiento de la productividad. El ciclo económico era un movimiento de equilibrio del sistema perfectamente coordinado. La teoría de los ciclos económicos reales se convirtió en el vehículo principal para el desarrollo de la teoría del DSGE. Esta teoría no atribuía ningún papel independiente al dinero o a las finanzas.

La coordinación en la teoría de los ciclos económicos reales era simplemente demasiado perfecta. La tradición de los Nuevos Clásicos, en términos generales, había soslayado los problemas de ajuste de corto plazo mientras que en una línea más o menos keynesiana se elaboraba un gran volumen de trabajo sobre estos temas. En los años que antecedieron inmediatamente a la crisis, los Nuevos Clásicos y los Nuevos Keynesianos comenzaron a converger en lo que se conoció como Nueva Síntesis Neoclásica. Los Nuevos Clásicos incorporaron algunas de las "fricciones" de los keynesianos mientras que éstos adoptaron el marco DSGE desarrollado por los primeros.

Nadie discutiría que hemos aprendido bastante en el intervalo de 50-60 años transcurrido entre la Vieja y la Nueva Síntesis Neoclásica. Un ejemplo es el acelerador financiero de Bernanke-Gertler así como el trabajo, relacionado con este acelerador, acerca del canal del crédito (e.g. Kiyotaki y Moore 1997) elaborado a partir de las contribuciones de Akerlof, Stiglitz y Greenwald sobre las implicaciones de la información asimétrica en los mercados de crédito. Otro ejemplo es el desarrollo de la teoría de la igualación de los mercados de trabajo por la cual Diamond, Mortensen y Pissarides, quienes construyeron su teoría a partir de McCall (1970), ${ }^{2}$ recientemente (y en forma justa) recibieron el Nobel. Estas contribuciones han desechado los conceptos primitivos y completamente ingenuos de mercados de capital

\footnotetext{
${ }^{2}$ Para otro ejemplo de trabajos importantes que se elaboraron a partir del de McCall, véase Ljungqvist y Sargent (1998).
} 
perfectos y mercados de trabajo perfectamente flexibles que recuerdo de mis días de estudiante hace 50 años. ${ }^{3}$

Aún cuando estos avances sobre cómo funcionan los mercados sin duda son importantes, nuestra comprensión acerca de cómo funciona una economía ha fallado en avanzar en un aspecto importante. La Vieja Síntesis Neoclásica, que veía a la economía como un sistema de equilibrio general estable obstaculizado por las fricciones de los salarios rígidos, extrajo la lección equivocada de la Gran Depresión y de la dramática deflación salarial que provocó en los Estados Unidos. La Nueva Síntesis Neoclásica nos ha llevado de regreso completamente a esta idea de que la economía es un sistema de equilibrio general estable con fricciones.

La Vieja Síntesis se equivocó en aquella época y considero que la Nueva Síntesis está equivocada hoy en día. No reconoce las inestabilidades que acechan en el sistema económico.

\section{COMPRENSIÓN INVERTIDA}

Por muchos años, Tony Lawson ha estado urgiendo a los economistas a que presten atención a los presupuestos ontológicos que ellos mismos formulan (véase Lawson 1997). Los economistas no le han hecho mucho caso, quizás porque pocos de nosotros sabemos lo que significa ontología. Esta rama de la filosofía hace hincapié en la necesidad de "comprender la naturaleza de la realidad" que constituye el objeto de estudio -y en la necesidad de adaptar nuestros métodos de investigación a esa naturaleza.

La teoría económica, podría argumentarse, ha entendido las cosas al revés. Hemos impuesto nuestros métodos preconcebidos sobre la realidad económica de tal modo que se ha distorsionado nuestra comprensión de la realidad. ${ }^{4}$

\footnotetext{
${ }^{3}$ Debe notarse, sin embargo, que las frecuentes alusiones a estos conceptos en forma de "fricciones" revelan que otras nociones mundanas de "mercados perfectos" todavía influyen en el pensamiento de la profesión.

${ }^{4}$ Cardoso y Palma (2009) también argumentan que hemos entendido la relación entre "método y disciplina" al revés. Su querella, sin embargo, no es tanto que al comprender las cosas en forma alrevesada hemos distorsionado la naturaleza de la realidad que se investiga, sino que hemos perdido toda definición de nuestro objeto de estudio, permitiendo que devenga en "generalmente cosas".
} 
Empezamos desde la elección óptima y creamos una imagen de la realidad que se adapte a ella. Transmitimos a nuestros estudiantes esta imagen distorsionada de cómo es el mundo insistiendo en que aprendan a percibir la disciplina a través de las lentes de nuestro método.

El mensaje central de la crítica que hace Lawson a la economía moderna es que la economía es un "sistema abierto", pero los economistas insisten en tratar a este sistema como si fuera "cerrado". Los experimentos controlados en las ciencias naturales crean cierres y de esta manera hacen posible la asociación no ambigua de "causa" y "efectos". Los macroeconomistas, en particular, nunca tienen el privilegio de tratar con sistemas que son cerrados en este sentido de experimento controlado.

Nuestras representaciones matemáticas tanto de la conducta individual como de la del sistema requieren el supuesto de cierre para que los modelos tengan soluciones determinadas. Lawson, por consiguiente, critica a la economía matemática y, de manera más general, al papel del deductivismo en nuestro campo. Aún los legos en ontología podemos reflexionar acerca de que tratar de deducir las propiedades de sistemas complejos muy grandes a partir de un pequeño conjunto de axiomas no es necesariamente una ambición razonable. Nuestros axiomas son, después de todo, mucho más endebles que los de Euclides.

\section{OPTIMIZACIÓN, EQUILIBRIO Y EXPECTATIVAS RACIONALES}

El ímpetu hacia el "cierre" en la macroeconomía moderna se deriva del compromiso con la conducta maximizadora concebida como los microfundamentos de la empresa. Los modelos de elección óptima vuelven a los agentes autómatas, entes carentes de "libre albedrío" y, así, los privan de poder elegir en cualquier sentido genuino. ${ }^{5}$ Los macrosistemas compuestos

\footnotetext{
${ }^{5}$ Es interesante comparar la postura de Lawson con la crítica de la economía neoclásica que realiza Spiro Latsis (1972). La literatura con la que Latsis discurrió hace cuarenta años no dio por supuesto la racionalidad sustantiva de los agentes. En vez de ello, la estrategia común para obtener predicciones teoréticas de comportamiento únicas fue "el determinismo situacional”, esto es, la creación de supuestos que estructuraran el problema de toma de decisiones de los agentes de tal modo que se les dejara sólo una "salida única".
} 
por tales autómatas excluyen la posibilidad de soluciones que puedan ser "desequilibrantes" en cualquier sentido significativo. Suceda lo que suceda, siempre están en equilibrio.

La extensión de este programa formal hacia el ámbito de la conducta "en el tiempo" requirió suponer que los agentes económicos poseían el conocimiento del futuro necesario para el cálculo de óptimos intertemporales. Las generaciones previas de economistas se mantuvieron distanciadas del supuesto de previsión perfecta (Hicks 1939 y 1985). En la década de los treinta, autores como Hayek, Lindahl y Hicks empezaron a tratar sistemáticamente el papel de las expectativas en el ciclo económico. Para ellos, las expectativas futuras influyen en las acciones presentes que crean el futuro -pero el futuro realizado rara vez se corresponde con lo que generalmente se espera. La economía es un sistema abierto en el sentido de Lawson. Los modelos de multiplicador y acelerador que se desarrollaron aproximadamente una década más tarde incorporaron esta misma perspectiva.

Es una cuestión práctica. El análisis de los mercados financieros de George Soros se inicia a partir de la concepción de que las creencias presentes acerca del futuro generan acciones que crean el futuro. Él llama a esta concepción reflexividad (Soros 2008). El inversionista que puede evaluar el sentimiento actual del mercado e inferir de qué manera puede producir un futuro distinto del que generalmente se espera puede obtener ganancias. Si interpreta incorrectamente al mercado o infiere equivocadamente, sufrirá una pérdida.

Las expectativas racionales son el caso especial, degenerado de la reflexividad donde el futuro que realmente ocurre siempre es uno de los posibles futuros derivados de la universalmente creída y verdadera distribución Gaussiana. Este supuesto hace a la economía un sistema cerrado. Se supone que los agentes poseen conocimiento (probabilístico) de una realidad objetiva - una realidad que han sido capaces de aprender. La lotería Gaussiana puede generar una ganancia o una pérdida. Pero la calidad de la información del inversor individual acerca del estado del mercado y su habilidad para extraer las inferencias apropiadas no tiene ninguna influencia en el resultado de la lotería. 
En el caso general de reflexividad, los agentes no sólo tienen que formarse expectativas acerca de una realidad objetiva futura sino que deben también formarse una opinión acerca de las expectativas de otros participantes del mercado. Una pequeña reflexión mostrará que detrás de semejantes expectativas de segundo grado acechan expectativas de grados aún más elevados - una casa de espejos en la que la realidad objetiva puede perderse por completo. ${ }^{6}$

\section{MUth Y LA TELARAÑA}

El teorema de la Telaraña que dio lugar al artículo original de Richard Muth (1961) titulado "Expectativas Racionales" surgió de la economía de la agricultura (Ezequiel 1938). Mostraba que si los agricultores escogían los acres para plantar la cosecha del próximo año tomando en cuenta el precio recibido por la cosecha del año en curso, el precio y el producto fluctuarían produciendo una secuencia alternante de ganancias no realizadas y, en consecuencia, pérdidas. ${ }^{7}$ Los agricultores que actuaban en la manera presupuesta por el modelo de la Telaraña siempre cometían errores costosos.

Muth demostró una manera en la cual los agricultores podían aprender a cambiar sus decisiones de siembra basándose en la expectativa estadística del precio del próximo año. Actuar sobre la base de esta expectativa racional eliminaría las fluctuaciones irracionales de la Telaraña. El procedimiento de inferencia estadística que propuso Muth no es necesariamente el modelo de aprendizaje más plausible para generar este resultado. ${ }^{8}$ iUn granjero muy arruinado en dos años consecutivos sólo podría cambiar su comportamiento con base en una muestra de tamaño dos!

\footnotetext{
${ }^{6}$ Este problema fue analizado detenidamente y en profundidad - sin tener grandes efectos sobre la profesión- por Phelps, Frydman, di Tata y otros en Frydman y Phelps (1983).

${ }^{7}$ Dada la elasticidad precio del producto y la elasticidad producto del precio de la demanda de la mayoría de los cultivos, el modelo de la Telaraña debería producir oscilaciones divergentes -haciéndolo aún menos plausible.

${ }^{8}$ Como Sydney Winter me comentó, la evidencia de la economía experimental es menos que esperanzadora con respecto a la capacidad de sujetos ordinarios para obtener inferencia estadística correcta.
} 
El punto más interesante acerca del caso Muth, sin embargo, es que las condiciones del problema son tales que hacen plausible que la solución racional pueda ser aprendida. Primero, hay una realidad objetiva que aprender que no está sujeta a los caprichos y debilidades del sentimiento del mercado, a saber, la distribución de las condiciones climáticas anuales y sus efectos sobre los retornos de la cosecha. Segundo, los ajustes de precio deben equilibrar la oferta y la demanda dentro del intervalo de tiempo fjo del año de la cosecha. ${ }^{9}$ La conjunción de estas dos condiciones crea una correspondencia de eventos entre el clima y el precio que, una vez aprendida, hace posible el comportamiento de expectativa racional. ${ }^{10}$

\section{SinCRONIZACIón}

La conjunción de estas dos condiciones anteriores, que hacen a las expectativas racionales un tanto plausibles, rara vez se lleva a cabo. La segunda condición, la existencia de un periodo de tiempo fijo dado sobre el cual la ley de la oferta y la demanda debe hacer su trabajo, merece algunos comentarios adicionales porque es un poco improbable que aparezca en la lista de supuestos a ser reexaminados cuando la teoría macroeconómica se encuentra en problemas.

En lugar de mercados de trigo o maíz, consideremos los mercados mundiales de petróleo o gas natural o algunos productos minerales. En estos últimos casos, no hay un "periodo natural" en el cual el precio equilibre la producción y el consumo. El precio no está determinado únicamente por "los fundamentales" de gustos y funciones de producción. Por supuesto,

\footnotetext{
${ }^{9}$ En los mercados para el ganado donde esta condición no aplica se observarán ocasionalmente unas cuantas rondas de las fluctuaciones de la telaraña. El término de Kaldor, "telaraña", se refiere a un dibujo en una pizarra. El nombre más antiguo de ello -"el ciclo del cerdo"- se refiere a fenómenos en un mercado real.

${ }^{10}$ Sin embargo sigue siendo un sistema abierto, por supuesto. La correspondencia entre el clima y el precio realizado puede cambiar por varias razones, tales como una disminución de los costos de transporte de una región de bajo costo -o, por supuesto, el calentamiento global. Más aún, no es necesariamente el caso, dado que se puede compilar una lista exhaustiva de posibles razones.
} 
generalmente se entiende cómo los cambios en los fundamentales afectarán a los precios en el tiempo. Pero en cualquier momento dado, las expectativas acerca de las expectativas de otros participantes del mercado juegan un papel inevitable en la administración de inventarios y en las decisiones de comprar o vender. El aprendizaje no elimina la heterogeneidad de las expectativas. Los comerciantes podrán no cometer errores sistemáticos pero continuarán cometiendo errores.

La heterogeneidad de las expectativas asociada con la falta de sincronización significa que habrá un rango de indeterminación dentro del cual el precio que despeja el mercado puede fijarse temporalmente. Este rango estará limitado. Habrá precios tan altos (o bajos) que la opinión predominante de los participantes del mercado los considerará insostenibles -y esto se convertirá en una profecía auto-cumplida. La amplitud de este rango de indeterminación variará en el tiempo. Prolongados periodos de tranquilidad, cuando la especulación tienda a estabilizar el nivel del precio dentro de un rango estrecho, contrastarán con episodios ocasionales de especulación extrapolando su tasa de cambio, lo cual ampliará enormemente este rango de indeterminación.

Cuando las condiciones del caso Muth no se cumplen, no tenemos derecho a suponer (1) que todos los participantes del mercado aprenderán la misma cosa o (2) que lo que aprendan será una verdad objetiva. Sin embargo, los economistas han tomado las expectativas racionales del contexto original de este caso especial y han trabajado con esto avanzando en cualquier dirección imaginable. En macroeconomía se ha aplicado a estados-espacio de dimensión infinita abarcando no sólo todos los mercados de futuros sino también una infinidad de mercados inexistentes. ${ }^{11}$

La representación matemática del sistema se cerró suponiendo que las expectativas racionales hicieron posible probar una variedad de proposiciones -tales como la equivalencia ricardiana y otros teoremas de inefectividad de política- que iban a contrapelo de la sabiduría convencional del momento.

\footnotetext{
${ }^{11}$ Incluso se afirma que las condiciones que despejan el mercado se sostienen en cada punto en el tiempo en modelos de tiempo continuo donde ni el precio ni la igualdad de las densidades de flujo de la oferta y la demanda tienen alguna interpretación sensata.
} 
Nada es más seductor para jóvenes reclutas en una disciplina particular que exponer las falsedades de la sabiduría convencional. La atracción de las expectativas racionales creció aún más por el reto de explicar cómo las cosas podrían salir mal en un mundo donde todos los agentes saben perfectamente lo que están haciendo. ${ }^{12}$ De este modo, las expectativas racionales generaron una buena dosis de fervor revolucionario en su tiempo. El entusiasmo ha decaído, aunque lentamente, toda vez que la teoría de las expectativas racionales se ha consolidado en una nueva ortodoxia considerada apropiada para el adoctrinamiento de las generaciones futuras.

\section{Descartando}

Las expectativas racionales conciben a la economía como un tren que viaja a través de un territorio markoviano cambiante. ¡Todos a bordo! Todos con el mismo equipaje mental cuidadosamente empacado. A intervalos constantes, predeterminados, el tren cambia - repentinamente- hacia una nueva vía elegida mediante un azar de lotería de feria. Las vías han sido colocadas de una buena vez (y no hay descarrilamientos). iNo es necesariamente la imagen más profunda de la condición humana! No obstante, renunciar a esta concepción de la naturaleza de una economía nos forzaría a modificar nuestros métodos. Aceptar que el futuro no puede conocerse con certidumbre, ni siquiera como una distribución de probabilidad, significa reconocer que estamos tratando con un sistema abierto. Y entonces la utilidad de muchas herramientas de esta teoría se pone en duda.

Los agentes en un sistema como éste tienen que adaptarse ${ }^{13}$ a eventos cuya probabilidad no han estimado correctamente $-\mathrm{o}$ que no pueden siquiera haber imaginado. Obviamente, la optimización intertemporal no puede ser

\footnotetext{
${ }^{12}$ Los incentivos son, por supuesto, la respuesta a las muchas variedades de este enigma. El enigma posterior viene entonces a explicar qué agentes racionales tienen incentivos estructurados para la gente de tal suerte que produzcan resultados no deseados. La respuesta más popular a ello ha sido "los políticos” o "el gobierno”. ¡La inocencia cínica de la economía moderna tiene una cierta inevitabilidad matemática al respecto!

13 Todas las Escuelas de Verano de la Universidad de Trento, de las cuales han habido 11 ediciones hasta ahora, han aparecido con el siguiente título general: "Dinámicas Económicas Adaptativas".
} 
entonces una representación "verdadera" de la conducta. El problema es que el tratar la conducta como adaptativa abre las puertas a toda suerte de conductas no lineales y uno no quisiera ver a la teoría macro reducida a algo más que un almacenamiento de cosas en la caja de juguetes de la dinámica de un sistema complejo.

Nuestras técnicas analíticas acostumbradas pueden aún ser útiles en el estudio del sistema abierto. En periodos de tranquilidad prolongada, los agentes están dispuestos a prestar atención a las tasas de sustitución intertemporal que confrontan y a extrapolarlas por algún tiempo en el futuro. Esto tenderá a desalentar la tendencia de la economía a fluctuar. Al menos suprimirá las oscilaciones de alta frecuencia. Esto es capturado por modelos de optimización intertemporal que pueden, de este modo, proveer aproximaciones de la conducta observada. Al hacer este uso de estos modelos, sin embargo, es mejor que recordemos que las condiciones de transversalidad en un horizonte de tiempo infinito no han de tomarse en serio. Cada burbuja que revienta a la postre prueba que la transversalidad es falsa. Entonces, qué tanto confiar en estos modelos se vuelve una cuestión de juicio -y esto no un juicio fácil.

En tiempos de volatilidad, la gente se encuentra obligada a reaccionar frente a los eventos del presente. Más aún, estos eventos frecuentemente interrumpirán planes que en ningún caso pudieron haberse llevado a cabo tiempo atrás. De este modo, la planeación intertemporal sobre periodos de tiempo significativos es vista como trivial. Esta es la lección en la que Daniel Heymann ha insistido desde hace tiempo, a saber, que los horizontes temporales de conducta varían endógenamente y que la teoría macroeconómica tiene que reflejar este hecho (Heymann y Leijonhufvud 1996 y Leijonhufvud 1997).

La volatilidad acorta los horizontes temporales. La conducta adaptativa miope genera dinámicas no lineales. Así, la volatilidad causa cortoplacismo que a su vez causa volatilidad. Esta ventana de retroalimentación positiva desaparece cuando la economía está en crisis. Heymann y yo discutimos en estos términos la desaparición de los mercados intertemporales y la volatilidad de los precios relativos en condiciones de elevada inflación. En las 
crisis de crédito recientes, hemos visto en operación un fenómeno similar cuando, a veces, importantes decisiones de política monetaria tuvieron que tomarse los domingos porque no podían esperar hasta el lunes.

Hacer la distinción entre condiciones tranquilas y volátiles, por sí misma no nos lleva muy lejos pero nos sirve para formular varias preguntas que no han recibido la atención que merecen por parte de los macroeconomistas. ¿Cómo difiere el comportamiento de los agentes individuales y el de la economía como un todo en momentos de recesiones agudas o de alta inflación respecto del de tiempos normales? ¿Qué tienen en común, si acaso, los dos extremos de la inestabilidad monetaria? ¿En qué sentidos son contrarios uno del otro? ¿Qué cosa conducirá a la economía allende la frontera de las condiciones tranquilas y en dirección de las condiciones volátiles? ¿Cómo podemos hacer que la economía regrese a su curso anterior?

\section{RESTRICCIONES PRESUPUESTARIAS}

Hasta ahora he discutido que, en un sistema "abierto", muchos precios estarán indeterminados (aunque dentro de ciertos límites), que el comportamiento económico tiene que entenderse como fundamentalmente adaptativo, que los horizontes temporales de conducta son variables, y que los conjuntos de mercados y de precios relativos pueden cambiar endógenamente. Los teóricos del equilibrio general están prestos a pensar que yo estoy haciendo un completo desorden de nuestra disciplina. Probablemente es correcto. Pero entonces, por supuesto, la realidad a veces es más desordenada. Para tomar en cuenta ese hecho, creo, debemos reconsiderar también el papel de la restricción presupuestaria en la teoría económica.

Hemos aprendido que una economía puede todavía mostrar una buena cantidad de orden estructurado, aunque sus participantes no sean súper inteligentes o no sean del todo racionales. Gary Becker mostró hace mucho tiempo (1962) que las curvas de demanda tendrían una pendiente negativa si los agentes fueran "irracionales" toda vez que la restricción presupuestaria los discipline. Más recientemente, Gode y Sunder (1993), en una contribución que ha generado bastante literatura, demostraron que los mercados alcanzarían un alto grado de eficiencia aún con "comerciantes con inteligencia 
cero". ${ }^{14}$ El mecanismo que asegura este resultado es de nuevo la restricción presupuestaria.

La restricción presupuestaria es uno de esos supuestos que parece escapar a la atención cuando el mundo real genera un ejemplo más de algo que contradice a la teoría económica. Janos Kornai es un raro ejemplo de economista que ha prestado atención a ello. Hace un cuarto de siglo, se centró en la restricción presupuestaria para tratar de entender las ineficiencias del socialismo. En un sistema donde los objetivos del plan frecuentemente violaban los requerimientos del punto de cierre para las empresas, la restricción presupuestaria era "suave". Kornai (1986) mostró que el mecanismo de precios no desempeñaría las funciones de asignación que generalmente le atribuimos en estas condiciones.

En las crisis económicas, las restricciones presupuestarias no son "suaves" sino que están "rotas" (Leijonhufvud 1998 y Heymann 2008). En crisis de deflación o depresión, las violaciones a la restricción presupuestaria están concentradas en el sector privado. En crisis de alta inflación o hiperinflación, es el soberano el que viola la igualdad en el valor de cambio. La prerrogativa del soberano de "crear dinero" significa que las consecuencias macroeconómicas difieren drásticamente de aquellas que surgen de las crisis financieras del sector privado.

El funcionamiento "normal" de una economía capitalista depende de que las restricciones presupuestarias sean vinculantes. Cuando ocurren violaciones grandes o generalizadas de la condición de igualdad en el valor de cambio, las dinámicas adaptativas de este sistema serán muy diferentes. Casi nada funciona como supone la teoría económica a menos que las restricciones presupuestarias sean realmente vinculantes. ${ }^{15}$ La teoría del equilibrio general estándar, aún en sus modernas variantes estocásticas dinámicas, no es particularmente útil cuando se violan las restricciones presupuestarias.

\footnotetext{
${ }^{14}$ Por supuesto, Becker al igual que Gode y Sunder, analizó casos simples de baja dimensión pero estoy dispuesto a creer que el verdadero significado de sus resultados puede también aplicarse de vez en cuando a dimensiones más altas. ¡Creer que siempre se aplican requiere, sin embargo, demasiada fe en la transversalidad!

${ }^{15}$ Los riesgos asegurables de moratoria pueden, por supuesto, manejarse con instrumentos estándares. Pero los riesgos que se cristalizan en una crisis financiera no han sido asegurados. En casos de
} 


\section{LA NATURALEZA DE UNA ECONOMÍA: INESTABILIDADES}

La imagen de una economía capitalista como un sistema de equilibrio general estable un tanto obstaculizado en su funcionamiento por "fricciones" es, creo, una guía inadecuada para las realidades con las que tenemos que enfrentarnos. Abrigar la posibilidad de serias inestabilidades está en la naturaleza de una economía. Es posible conjeturar acerca de las propiedades cualitativas de sus dinámicas. ${ }^{16}$ Las inestabilidades más dañinas surgen de las violaciones a la restricción presupuestaria. Lo mejor es comenzar distinguiendo entre violaciones a la restricción presupuestaria por parte del sector privado y por parte del soberano.

Imaginemos primero una representación estado-espacio del sector privado dividida en tres regiones. En la primera región (M1) del espacio el sector del mercado mostraría un comportamiento "normal". Los controles de retroalimentación negativos dominan en todos los mercados y las políticas de estabilización, en el sentido convencional, no son útiles. En la segunda región (M2), se producen retroalimentaciones adaptativas desestabilizadoras (positivas) pero están limitadas muy estrechamente. Algunos ejemplos son el multiplicador keynesiano y el acelerador financiero. La economía discurre a través de ciclos económicos. Las políticas monetaria y fiscal pueden ser útiles para cambiar la liquidez o afectar directamente la demanda agregada. En la tercera región (M3), se encuentran las inestabilidades verdaderamente peligrosas tales como avalanchas de moratoria. En alguna parte de esta región se encuentra el "hoyo negro" de una catástrofe Fisheriana de deuda y deflación.

En el proceso que puede propulsar a la economía de la Región M2 a la Región M3, el apalancamiento (Geanakoplos 2010 y Leijonhufvud 2009a) juega un papel crucial. La acumulación lenta de apalancamiento en la economía incrementa la conectividad de la red (Leijonhufvud 2009b) de deudas

\footnotetext{
contratos contingentes, decidir qué constituye el incumplimiento del contrato puede ser un asunto complicado. Para una discusión de esto véase Heymann (2008:76).

16 Este esquema de regiones estado-espacio de 2x3 fue tomado de Leijonhufvud (2009c). Este artículo elabora un poco la "hipótesis de corredor" que Leijonhufvud propuso aproximadamente hace 40 años.
} 
y títulos y se combina con la subyacente discrepancia del vencimiento de los títulos haciendo al sistema más frágil. Cuando eventualmente el sector financiero se desplaza hacia el desapalancamiento, la conectividad del sistema propiciará que diversos mecanismos de compensación-desviación se "sincronicen".

El estado-espacio para un sistema gobernado por las finanzas del soberano nos daría una primera región (S1) con superávit presupuestarios o déficit sostenibles creíbles. Una segunda región (S2) tiene déficit que engendra la inflación esperada con una correspondiente prima de Fisher sobre la tasa de interés. En esta región, sin embargo, el gobierno conserva una parte sustancial de control de la situación. La tercera región (S3) del lado del público es una zona de alta inflación donde se ha perdido el control. Las expectativas de inflación de corto plazo responden con gran celeridad a las acciones del gobierno, mientras que las expectativas de más largo plazo están muy mal definidas como para apoyar mercados intertemporales de cualquier clase. El "hoyo negro" en este lado es, por supuesto, la verdadera hiperinflación (Heymann y Leijonhufvud 1996 y Leijonhufvud 1998).

En esta parte, el principal mecanismo que lleva al sistema de la Región S2 a la Región S3 es el efecto Olivera-Tanzi (Olivera 1967 y Tanzi 1977) que, por supuesto, es bien conocido por todos los lectores argentinos.

Un punto adicional en esta parte del artículo. Principalmente, la macroeconomía ha tratado con relaciones entre variables de flujo. La suposición tácita es que las hojas de balance se han desarrollado más o menos "en la trayectoria adecuada". ${ }^{17}$ En las regiones $3 \mathrm{M}$ y 3S, las hojas de balance están en un gran "desequilibrio" y esto domina la dinámica del sistema (Koo 2003). En recesiones severas, los principales problemas son los intentos del sector privado para "desapalancar" y así restablecer las hojas de balance a un estado razonablemente saludable. Cuando la inflación es alta, la completa destrucción del sector financiero es la que impide que la economía crezca porque el crecimiento no puede ser financiado.

\footnotetext{
${ }^{17}$ Esto también vale, yo diría, para la Teoría General y es la razón principal no acrítica para "regresar a Keynes", como algunos pueden haber argumentado en respuesta a la presente crisis.
} 


\section{Doble Peligro}

Estamos acostumbrados a pensar que las depresiones y las inflaciones altas son extremos opuestos de un espectro de posibles condiciones macroeconómicas. Pero unas son provocadas por insolvencias del sector privado y las otras por insolvencias del gobierno. La primera condición, desafortunadamente, no excluye a la otra (Heymann 2008).

Los recursos del gobierno deben ser utilizados para sacar al sector privado de una grave recesión o depresión. Los recursos deben transferirse del sector privado al público para controlar la inflación alta. Pero si las finanzas de un sector ya están deterioradas cuando el otro sector experimenta problemas, puede haber opciones de política no muy agradables. La historia financiera de América Latina registra varios episodios donde, para evitar depresiones, los gobiernos han nacionalizado los activos malos de sus sistemas bancarios -y así la política ha socavado las finanzas públicas para lanzar a la economía a un estado de alta inflación. ${ }^{18}$

En el presente, como todos sabemos, las políticas de los Estados Unidos y de Europa están en conflicto. En ambos lados del Atlántico, los gobiernos enfrentan un doble peligro: una probabilidad significativa de verse condenados si hacen algo y de verse condenados también si no hacen nada. Alemania, que prospera hasta ahora sobre la base de sus exportaciones, está forzando a la Eurozona a dar prioridad a la estabilidad financiera de más largo plazo de los gobiernos. El nuevo gobierno del Reino Unido se está moviendo en la misma dirección. Hasta ahora la administración estadounidense ha estado dispuesta a aumentar su déficit para inducir al sector privado a un nivel de empleo más satisfactorio.

Ambas partes se encuentran jugando el mismo juego de doble peligro pero no perciben las opciones de la misma manera. La principal diferencia puede ser que las autoridades de los Estados Unidos han aprendido que la volatilidad en la economía mundial incrementa, en el mediano plazo, la

\footnotetext{
${ }_{18}$ Aprendí esta lección de Daniel Vaz (1999) y frecuentemente he hecho alusión a su trabajo desde entonces.
} 
demanda de activos denominados en dólares aún si el balance fiscal del gobierno federal es cada vez más precario en el largo plazo. Ningún otro país disfruta de este beneficio - ni de las tentaciones que vienen con ello.

Europa y los Estados Unidos, actuando con propósitos cruzados, nunca presagiaron bien la recuperación económica de lo que solía conocerse como "los poderes occidentales". Ahora la victoria republicana dicta el fin de un renovado estímulo del gasto. Esto nos deja con los esfuerzos redoblados de "relajación cuantitativa" por parte de los bancos centrales. Esa estrategia, me temo, conlleva el riesgo de ponernos en triple peligro.

En esta situación, los economistas tenemos que estar conscientes acerca de los límites de nuestro conocimiento. Algo sabemos acerca de lo que se necesita hacer para detener una inflación alta o recuperarnos de una recesión aguda. ${ }^{19}$ No pienso que tengamos conocimiento muy confiable acerca de cómo jugar estos juegos de doble - o triple- peligro.

\section{Distribución}

La macroeconomía moderna se ha apoyado fuertemente en la función de producción neoclásica y ha aceptado la teoría de la productividad marginal de la distribución que deriva de ella. Los herederos intelectuales de Adam Smith, que creen que el crecimiento económico surge en gran parte de la creciente división del trabajo, considerarán esto como un sin sentido insustancial. Si la estructura productiva de una economía muestra rendimientos crecientes en muchas de sus dimensiones, no puede ser cierto que a los factores de producción en general se les paguen sus respectivos productos marginales.

Cualquier posición que uno adopte sobre esto, sin embargo, obviamente es un asunto que está dentro de la teoría económica del equilibrio, y esa rama de nuestra disciplina no tiene mucho que decirnos acerca de las consecuencias distributivas de procesos altamente inestables. Las principales consecuencias no son cambios en las ganancias productivas derivadas de los esfuerzos productivos, sino redistribuciones de la riqueza. En última instancia, resultará

\footnotetext{
${ }^{19}$ Pero si los economistas hubieran sido capaces de estar a la altura de sus pretensiones de conocimiento, Japón no hubiera tenido que languidecer en recesión tantos años.
} 
que las dotaciones habrán sido reorganizadas pero el proceso de aclarar "quién es dueño de qué" es largo, prolongado y muy costoso.

La incidencia distributiva de una gran crisis financiera no tiene sentido para la gente común. Las ganancias y pérdidas que experimentan y observan no se relacionan con las reglas mediante las cuales han conducido sus vidas. Las políticas de emergencia requeridas para detener a medio camino el colapso de un castillo de naipes financiero -que en última instancia los contribuyentes fiscales comunes tienen que pagar- tampoco tiene sentido para ellos. La difundida desorientación y la rabia suministran un ambiente favorable para la demagogia y la búsqueda de chivos expiatorios de grupos étnicos, de inmigrantes, o de naciones extranjeras. La pérdida de un sentido de solidaridad social hace crecientemente difícil el acopio de consenso suficiente para las políticas que confronten la crisis. No hace falta decir que los consensos entre naciones son aún más difíciles de conseguir.

Vale la pena discutir en más detalle un ejemplo de estos problemas distributivos, no porque sea de gran importancia cuantitativa, sino porque ilustra el tipo de problemas que salta a la vista en la inestable Región 3 del sistema estado-espacio. También ilustra cómo algunos de estos problemas han escapado al escrutinio público.

En los días de apogeo del monetarismo, la neutralidad del dinero se aceptó ampliamente aunque también se reconoció que el dinero no era completamente súper-neutral. La neutralidad significaba que la política monetaria no tendría consecuencias distributivas, excepto aquellas que surgieran de las fallas ocasionales de la gente al predecir correctamente la tasa de inflación. Hoy, en los Estados Unidos, el Sistema de la Reserva Federal presta reservas al sistema bancario a una tasa de interés esencialmente igual a cero. Los bancos usan estas reservas para comprar bonos del Tesoro más o menos a 4\%. Este es un gran subsidio al sistema bancario que al final de cuentas pagarán los contribuyentes fiscales -pero ni el subsidio ni los pasivos tributarios han sido votados por el Congreso. Más aún, la política de tasa de interés cero del Banco Central reduce la tasa de interés disponible para los ahorradores hasta una pequeña fracción de 1\%. Al mismo tiempo, los bancos apalancan su capital por un factor de 15 o más, ganando de esta manera una tasa verdaderamente excepcional sobre su capital al comprar 
bonos a 4\% con dinero sin costo de la Reserva Federal. Los banqueros de Wall Street pueden otra vez obtener los beneficios extraordinarios a los que estaban habituados en los buenos viejos días antes de la crisis ${ }^{20}$ y que supuestamente son bien merecidos por el genio requerido para llevar a cabo esta operación. Estas ganancias extraordinarias son, en efecto, transferencias de los ahorradores más viejos que no pueden encontrar una alternativa más segura para sus fondos de jubilación.

\section{Conclusión}

Se han enfatizado dos temas en este artículo:

- El primero es que tenemos que pensar a la economía como un "sistema abierto" en el sentido ontológico de Tony Lawson.

Esto requerirá que adaptemos nuestros métodos a la naturaleza de una economía - para cambiar la manera en la que hacemos economía.

- El segundo es que la economía no es globalmente estable sino que entraña inestabilidades.

Algunas de estas inestabilidades están limitadas muy estrechamente, pero otras son potencialmente catastróficas para el bienestar económico y pueden ser sumamente dañinas para la solidaridad social y para la estabilidad política.

La macroeconomía, en los años que condujeron a la reciente crisis, se había convertido en una disciplina técnicamente exigente y naturalmente fue dominada por gente que era buena en ese tipo de cosas. Pero a menos que uno se sintiera muy orgulloso y alegre con estos aspectos técnicos, la macroeconomía también habría devenido mortalmente aburrida.

La crisis debió de habernos curado de la "presunción de conocimiento" (Caballero 2010) -de la ilusión de que entendíamos muy bien los problemas de la inestabilidad macroeconómica y de que los habíamos resuelto todos para la satisfacción general. Una vez curados de esta presunción -lo que

\footnotetext{
${ }^{20}$ También pueden usar estas ganancias para devolver el dinero comprometido por el gobierno para salvarlos de la insolvencia. Y el gobierno entonces alegará que en última instancia el rescate no le costó a los contribuyentes fiscales.
} 
equivale a decir, una vez conscientes de nuestra ignorancia- podemos ver que la macroeconomía plantea un gran número de importantes preguntas para las cuales mi generación no proporcionó buenas respuestas.

Esto debiera hacer de nuestra disciplina algo lleno de entusiasmo intelectual para quienes son unas décadas más jóvenes.

\section{REFERENCIAS}

Becker, G., "Irrational behaviour and economic theory", Journal of Political Economy, vol. LXX(1), 1962, pp. 1-13.

Caballero, R.J., "Macroeconomics after the crisis: time to deal with the Pretenseof-Knowledge Syndrome", National Bureau of Economic Research (NBER), Working Paper no. 16429, 2010.

Cardoso, J.L. y N. Palma, “The science of things generally?”, en A. Witztum y F. Cowell (eds.), Lionel Robbins's Essay on the Nature and Significance of Economic Science -75 th Anniversary Conference Proceedings, Londres, London School of Economics, STICERD, 2009, pp. 387-402.

Ezekiel, M., "The cobweb theorem”, Quarterly Journal of Economics, vol. 52, 1938, pp. 255-280.

Friedman, M., "The role of monetary policy", American Economic Review, vol. 58(1), 1968, pp. 1-17.

Frydman, R. y E.S. Phelps (eds.), Individual Forecasting and Aggregate Outcomes: "Rational Expectations" Examined, Cambridge, Cambridge University Press, 1983.

Geanakoplos, J., "The leverage cycle”, en D. Acemoglu, K. Rogoff y M. Woodford (eds.), NBER Macroeconomic Annual 2009, Chicago, Chicago University Press, 2010.

Gode, D.K. y S. Sunder, "Efficiency of markets with zero intelligence traders: market as a substitute for individual rationality", Journal of Political Economy, vol. CI(1), 1993, pp. 119-137.

Heymann, D., "Macroeconomics of broken promises", en R. Farmer (ed.), Macroeconomics in the Large and the Small, Cheltenham, Edward Elgar, 2008.

Heymann, D. y A. Leijonhufvud, High Inflation, Oxford, Oxford University Press, 1996. Hicks, J.R., Value and Capital, Oxford, Oxford University Press, 1939. -, Methods of Dynamic Economics, Oxford, Oxford University Press, 1985.

Kiyotaki, N. y J. Moore, “Credit cycles", Journal of Political Economy, vol. 105(2), 1997, pp. 211-248. 
Koo, R.C., Balance Sheet Recession: Japan's Struggle with Unchartered Economics and its Global Implications, Singapore, Wiley, 2003.

Kornai, J., "The soft budget constraint”, Kyklos, vol. 39(1), 1986, pp. 3-30.

Ljungqvist, L. y T.J. Sargent, "The European unemployment dilemma”, Journal of Political Economy, vol. 106(3), 1998, pp. 514-50.

Latsis, S.J., "Situational determinism in economics", British Journal for the Philosophy of Science, vol. 27, 1972, pp. 51-60.

Lawson, T., Economics and Reality, Londres, Routledge, 1997.

Leijonhufvud, A., "The Wicksell connection: variations on a theme”, en Information and Coordination: Essays in Macroeconomic Theory, Nueva York, Oxford University Press, 1981, pp. 131-202.

_- "Keynesian e conomics: past confusions, future prospects", en A. Vercelli y N. Dimitri (eds.), Macroeconomics: A Survey of Research Strategies, Oxford, Oxford University Press, 1992, pp. 16-37. Reimpreso en A. Leijonhufvud, Macroeconomic Instability and Coordination: Selected Essays, Cheltenham, Edward Elgar, 2000, pp. 33-51.

—, "Macroeconomic complexity: inflation theory", en W.B. Arthur, S.N. Durlauf y D.A. Lane (eds.), The Economy as an Evolving Complex System II, Nueva York, Addison Wesley y the Santa Fe Institute, 1997. -, "Two types of crises", Zagreb Economic Journal, diciembre, 1998. -, "Two systemic problems", CEPR Policy Insight, núm. 29, enero, 2009a. , "Curbing instability: policy and regulation", CEPR Policy Insight, núm. 36, julio, 2009b.

_-, "Instabilities", Trabajo presentado en la inauguración de la Conferencia INET, Cambridge, Reino Unido, abril 8-11 de 2010, 2009c.

McCall, J.J., "Economics of information and job search", Quarterly Journal of Economics, vol. 84(1), 1970, pp. 113-26.

Muth, J.F., "Rational expectations and the theory of price movements", Econometrica, vol. 29(6), 1961.

Olivera, J.G.H., "Money, prices and fiscal lags: a note on the dynamics of inflation", Banca Nazionale del Lavoro Quarterly Review, vol. 20, 1967, pp. 258-267.

Soros, G., The New Paradigm for Financial Markets: The Credit Crisis of 2008 and What It Means, Nueva York, Perseus Books, 2008.

Tanzi, V., "Inflation, lags in collection, and the real value of tax revenue", IMF Staff Papers, vol. 24, 1977, pp. 154-167.

Vaz, D., "Four banking crises: their causes and consequences", Revista de Economía, vol. 6(1), 1999, pp. 29-346. 